\title{
Review of Cement Concrete Pavement of Noise Reduction
}

\section{Method}

\author{
Hongliang $\mathrm{Tao}^{1}$, Chen $\mathrm{Chen}^{1}$, Ping Jiang ${ }^{2, *}$ and Shuaihong Huang ${ }^{2}$ \\ ${ }^{1}$ Taffic Planning and Design Institute of Yunan Province, Kunming City, Yunnan Province, China \\ ${ }^{2}$ Shaoxing University, Shaoxing City, Zhejiang Province, China \\ Corresponding E-mail: jiangping1119@163.com
}

\begin{abstract}
In recent years, with the rapid development of the economy, a sharp increase in car ownership in China, the rapid increase in cities traffic volume. Meanwhile, the effect on the normal life, work, study, rest and environment of residents along grows increasingly fierce both in extent and scope. Cement Concrete Pavement noise reduction measures seem more urgent. In this paper, introducing the current commonly used cement pavement noise reduction measures, and discussing the research and development direction of cement concrete pavement noise reduction measures.
\end{abstract}

Keywords. Traffic engineering, Cement concrete pavement, Noise causes, Noise reduction measures.

\section{Introduction}

Highway is the lifeline of the national economy, is the image of regional development, defense and stable basis, and only pavement keep long-term smooth condition, in order to ensure that the vehicle quickly and safely on the roads. According to the survey: noise has a negative impact on the human psychology and the body at the same time, particularly harmful to the nervous system and cardiovascular system; noise can damage children's brains, children who long-term in a noisy environment, their intellectual development than in the quiet environment for children approximately $20 \%$ less [1]. With the development of transportation construction, as of 2013, China's total mileage has more than 4 million km, cement concrete pavement with a mechanical strength, high load capacity, less prone to plastic deformation, etc., is one of the main basic form of road pavement. However, due to its stiffness, traffic noise is quite obvious. With more and more attention to environmental engineering, as well as comfort and road safety and environmental protection have become increasingly demanding. Cement concrete pavement noise pollution problem of environmental pollution has become most concerned of residents. Therefore, taking effective measures to reduce traffic noise pollution to maintain a good acoustic environment along the road has been a priority [2].

\section{Cement concrete pavement noise generation mechanism}

The noise of vehicles driving on the cement concrete pavement can be divided into: First, noise emitted by automobiles, mainly in the high-power motor vehicle engine noise for most, with its main class of vehicle, usually in the engine of the vehicle can be equipped with mufflers effectively reduce the noise. Second, car horn, mainly produce emergencies when the car in motion the process to remind drivers to produce, increased by about $8 \mathrm{~dB}$ overall car tweeter automobiles can emit noise, general urban road approach can be used to reduce the noise control horn produced; Third, vibration of the interaction between tire and cement pavement produce the noise. This process of the generation of noise is very 
complicated, but mainly due to the air pump at high speed between the tire and the road surface and the vibration effect of the tire[3]. Tires at high speed rolling process, road to the vibration of the tyre tread and sidewall effect caused by noise, there are shock vibration noise, slip vibration noise, rehabilitation vibration noise and road vibration noise [4]. Generally, the proportional to the size of the noise and driving speed of the car. When all types of car speed exceeds $55 \mathrm{~km} / \mathrm{h}$, tire and road noise generated by friction has become a major part of the road traffic noise. In addition, the strength of road traffic noise is also affected by the road line, slope and roadbed height and many other factors [2].

Reduce the noise generated by the interaction with the cement pavement concrete pavement and tire is the main method of noise reduction. Because this type of noise generation related with tire tread and road construction and road surface texture. Therefore, the study of the concrete pavement noise reduction measures along these two basic ideas that optimize the tire tread expand and optimize the structure of concrete pavement and road textures, this article will optimize the texture of the pavement structure and road noise reduction measures used to introduce the cement pavement.

\section{Noise reduction measures pavement structure}

\subsection{Pavement Material Type}

\subsubsection{Porous concrete pavement}

The main reason is that porous concrete reduce noise is there is a wide gap in internal material structure. When sound waves enter the internal structure of the material, caused the air movement in the pores, and pore formation of solid body friction, due to the effects of viscosity and thermal conductivity, a portion of the sound energy into heat energy consumed; sound waves reflected by the rigid wall after the material returns to its surface, a portion of the acoustic wave back to the air permeable and partly reflecting back into the interior material, this process is repeated propagation of sound waves, is the energy dissipation constant conversion process, so the material on the "absorption" of the section sound energy [5].

\subsubsection{Rubber Concrete Pavement}

Rubber powder particles of cement-based composite materials is a new kind of impact resistant toughened functional materials developed in recent years, because of its existing large strain and stretch the limits of effects and impact resistance, low modulus, but also has excellent mechanical performance and good noise reduction, therefore, as the city noise insulation materials and impact-resistant bumper isolation member, it will have broad application prospects in municipal engineering neighbourhood.

The higher driving speed, the more significant of noise performance of rubber cement pavement. Rubber cement rubber particles uniformly distributed in the road pavement layer to form a multi pore characteristics, can weaken the sound waves produced between tire and road surface due to the "two sides effect", Simultaneously added such that the rubber particles in the surface acoustic wave transmitting and reflecting some of the noise at the time of the process through the rubber particles, sound waves into mechanical energy of vibration is weakened rubber particles, thus weakening the secondary noise transmission [6].

\subsubsection{Exposed Aggregate Concrete Pavement}

Exposed aggregate concrete pavement can be used in new construction, renovation or reconstruction of cement [7]. Exposed concrete pavement because of random convex surface of the aggregate, sound waves and pressure waves dissipate on their own in the space under the tread, $7 \mathrm{~dB}$ lower than the old concrete pavement noise, and its surface is not easy to form a film of water, even in the rain can be maintain a high skid resistance. After treating the surface of exposed aggregate concrete pavement, rough road can form diffuse, eliminate or mitigate the diffuse reflection [1]. 


\subsubsection{Asphalt Overlay Low Noise Pavement}

Asphalt overlay low noise pavement to take effective measures against noise between the tire and the pavement, which a pavement structure type for the purpose of reducing noise. Porous asphalt pavement, or called permeable (or drainage) roads. It refers to the ordinary asphalt or cement concrete pavement or other paved pavement layer has a high porosity layer of asphalt mixture [8]. Its noise reduction mechanism is surface voids' sound absorption. In addition to absorb the noise of the engine and transmission parts radiation to the pavement outside, but also can absorb reflected noise road surface is reflected back through the car chassis road tire noise and other interfaces [9].

\subsubsection{Diamond Polishing Concrete Pavement}

Diamond grinding is a new technology of cement concrete pavement surface repair measure function refers to the use of fine-pitch diamond blade cutting abrasive concrete pavement, grinding away the surface of 4-6mm thickness, a certain vertical grain structure. The main purpose is to eliminate defects in the road surface, improving cement concrete pavement surface features and reduce road noise [7].

\subsection{Road Surface Texture}

\subsubsection{Picking}

Picking is instructed to produce shallow texture in concrete road surface to slow the pavement wear, thus reducing road noise. However, road surface texture produced by the technologies of nap is relatively, worn between tires and the road surface interaction will often reduce the feature of concrete pavement. Through the reasonable design improve the wear resistance of concrete pavement, to ensure that there is enough road surface features during the service period, so achieve the purpose of reducing road noise.

\subsubsection{Groove}

Groove is generated shallow channels on the surface of the concrete pavement, to increase the drainage capacity and the friction under the condition of slippery roads in varies weather conditions, but also has reduced road noise [7]. But the noise reduction effect on medium and heavy trucks of grooving roads.

\subsubsection{Pressure Trough}

Pressure trough, lateral pressure trough is the most common kind of concrete pavement surface texture forming method is a durable, high friction resistance and low-cost approach. Deep macro structure produced by lateral pressure trough can reduce the thickness of the film of water on rainy roads, reducing the potential threat of skidding in rainy wet road. But the noise generated by the interaction of the tire and the road surface on the lateral pressure trough was the largest.

\section{Other noise reduction measures}

\subsection{Setting The Sound Barrier}

Noise barrier mainly through the sound absorption and sound insulation to reduce noise, absorption is mainly by increasing the sound-absorbing material in the sound barrier to achieve, and insulation is mainly dependent on increasing the propagation distance of noise to achieve, in the process of through a few noise baffle of the sound barrier, the noise has formed a reflective, so as to continuously reduce its strength. So the noise reduction effect of sound barrier some extent depends on the height and length of the sound barrier [1]. 


\subsection{Set Green Belt}

Setting green belt refers to the land within the scope of the road for the green bar area. By using acoustic methods of setting green belt to protect the ecological environment, this noise reduction method is a long-term, sustainable improvement of ecological environment, the role of environmental protection is very prominent. Use of greening ecological protection measures to reduce noise is one of the effective and environmental protection measures, In the actual road construction, planting patterns of green belt and noise protective effect has a strong correlation.

\section{Looking to cement pavement noise reduction measures}

\subsection{Noise Reduction Materials Research}

All of the above concrete pavement noise reduction measures are based on hydraulic concrete materials, by changing the structure of the road to achieve the purpose of noise reduction[10]. With the rapid development of society, the use of cars is growing, more and more waste tires were produced, and now the repeated utilization of waste tires is very low, used tires, black pollution has become increasingly serious environmental problems. So based on the basic principle of exposed concrete pavement noise reduction, the rubber particles used in cement concrete pavement[11], not only can be clean utilization of scrap tire, save resources, also can improve the performance of the cement concrete.

\subsection{Research and Development of Road Noise Test System}

Since there is no test system to simulate actual road noise, and therefore cannot form a scientific research methods road noise, such as the use of the tire falling to simulate the impact of car tires on cement concrete pavement used by Hansen, Dong Yuming, And collecting the generated noise, to evaluate the noise reduction feature pavement materials [3]. Perfect noise test system can simulate different axle load and driving speed, and has the ability to obtain noise data between tire and road accurately.

\section{Conclusion}

The research for cement pavement noise reduction measures can create a better living and working environment for residents along, for now, noise reduction measures can be divided into the pavement structure and other noise reduction measures, Future noise reduction of cement pavement can around the development of new noise reduction materials, such as rubber aggregate concrete pavement and development of road noise test system.

\section{References}

1. Yang Xiang-tao: Analyse Cement concrete pavement noise reduction technology, Technology Guide Vol.2 (2012), p.397 .

2. Zhuo Yong-hong: Introduction to highway traffic noise and noise reduction measures, Highways \& Transportation in Inner Mongolia Vol.4 (2013), p.17-18.

3. Han Sen, Dong yu-ming, Chen hai-feng: Noise Reduction Performance of Exposed-aggregate Cement Concrete Pavement, Journal of Traffic and Transportation Engineering Vol.3 (2005), p.32-34 .

4. Shan Yong-ti, Li Zhu-ming, Yuan Wei-ming, Zhu Wei-hong: Study on the Noise Reduction Effect by Monitoring of the Low-noise Pavement, Environmental Monitoring and Forewarning Vol.3(2011), p.45-50.

5. Jing Lu-bo, Tian Bo, Liu Ying, Niu Kai-min. Research on Noise Reduction Properties of Porous Cement Concrete Pavement, Highway Vol.7(2010), p.75-79. 
6. He Zhuang-bin, Qin Feng: Mechanical Characteristics and Noise Reduction Performance of Rubberized Concrete, Highway Engineering Vol.5 (2013), p.128-131.

7. Yu Jie, Wang Jun-jie, Zhang Peng: Cement Concrete Pavement Noise Reduction Measures, North Traffic Vol.4 (2012), p.22-24.

8. Wang Tian-yang, Li Jing-yang: A Research on the Urban Noise Reduction Road Surface, Communications Science and Technology Heilongjiang Vol.8 (2011),p.50-52.

9. Wu Xin-feng: Types of Low Noise Pavement and their Applicability Analysis, Transpo World Vol.5-6 (2012), p.196-197.

10. Liu Ying, Tian Bo, Niu Kai-min: Skid-resistance and Denoising Properties of Cement Concrete Pavement with Different Surface Texture, Journal of Highway and Transportation Research and Development Vol. 29(2012), p.28-33.

11. Li Bo, Han Sen: Some Methods on Concrete Pavement Noise Reduction, Noise and Vibration Control Vol.3 (2008), p.127-130. 